\title{
List of Papyri and Manuscripts
}

\section{Papyri and ostraca}

Cairo, Coptic Museum, inv. $6569+6570+$ 657123

Grand Haven, Scriptorium, Mississippi

Coptic Codex II (+ P. Bodmer XXII) 31

n. $87,33,56$

O. Berlin. P. 12509103

O. Vindob. K 187105

O. Vindob. K 593104

Oslo, Schøyen Collection, MS 193 (+ P. Chester Beatty 2026) 31 n. 89, 55

P. Amh. I 1 XIV, 127

P. Ant. I 19 78, 94

P. Ant. II 58 78, 94

P. Ant. II 6578

P. Barc. inv. 157b $47+$ n. $120,48+$ Fig. 27 + n. 121

P. Beatty IV 77

P. Beatty XII 20

P. Berol. inv. 325937

P. Berol. inv. 5003 (+ P. Cairo 140 and P. Gen. IV 158) 46

P. Berol. inv. 5011 10, 122

P. Berol. inv. 6794 77, 80, 93, 94, 96, 124

P. Berol. inv. 9722134 n. 291

P. Berol. inv. 10566 (+ P. Berol. inv. 10558 and 10559) 20

P. Berol. inv. 10558 (+ P. Berol. inv. 10566 and 10559) 20

P. Berol. inv. 10559 (+ P. Berol. inv. 10566 and 10558) 20

P. Berol. inv. 11754 (+ P. Berol. inv. 21187) 147 n. 342

P. Berol. inv. 13262 (+ P. Berol. inv. 21228) XIV

P. Berol. inv. 13273136

P. Berol. inv. 13418 XIV

P. Berol. inv. 13929 (+ P. Berol. inv. 21105)

$70,76,80,96,124$

P. Berol. inv. 13932 (+ PSI II 126) 156

P. Berol. inv. 13994 22, 23

P. Berol. inv. 16353 76, 80, 96, 122, 124

P. Berol. inv. 21105 (+ P. Berol. inv. 13929)

70 Fig. 35, 76, 80

P. Berol. inv. 21187 (+ P. Berol. inv. 11754) 147 n. 342

P. Berol. inv. 21228 (+ P. Berol. inv. 13262) XIV

P. Berol. inv. 21900149

P. Bingen 1961 n. 142

P. Bodmer II (+ P. Köln V $214+$ P. Chester Beatty Ac. 2555) 32 n. 93, 55

P. Bodmer III 31 n. 89, 35, 55

P. Bodmer IV (+ P. Bodmer XXV + P. Bodmer XXVI + P. Köln VIII 331+ P. Duke inv. 775) 43, 44 Fig. 24, 45 + Fig. 25, 46 Fig. 26, 47 n. 117, 54, 55, 56

P. Bodmer V (+ P. Bodmer X, XI, VII, XIII, XII, XX, IX, VIII) [Miscellaneous Codex] 31, 35, 37 n. 106, 38 Fig. 18, 55

P. Bodmer VII (+ P. Bodmer XI, X, V, XIII, XII, XX, IX, VIII) [Miscellaneous Codex] 37 n. 106, 42 n. 112, 43 n. 113, 55

P. Bodmer VIII (+ P. Bodmer IX, XX, XII, $\mathrm{XIII}, \mathrm{VII}, \mathrm{XI}, \mathrm{X}, \mathrm{V}$ ) [Miscellaneous Codex] 37 n. 103 ] n. 106, 42, 43 Fig. 23, 55

P. Bodmer IX (+ P. Bodmer XX, XII, XIII, VII, $\mathrm{XI}, \mathrm{X}, \mathrm{V}, \mathrm{VIII}$ [Miscellaneous Codex] 37 n. 106, 40, 42 Fig. 22, 55

P. Bodmer X (+ P. Bodmer V, XI, VII, XIII, $\mathrm{XII}, \mathrm{XX}, \mathrm{IX}, \mathrm{VIII})$ [Miscellaneous Codex] 37 n. 106, 38, 39 Fig. 19, 43, 55

P. Bodmer XI (+ P. Bodmer X, V, VII, XIII, $\mathrm{XII}, \mathrm{XX}, \mathrm{IX}, \mathrm{VIII})$ [Miscellaneous Codex] 37 n. 106, 55

P. Bodmer XII (+ P. Bodmer XIII, VII, XI, X, $\mathrm{V}, \mathrm{XX}, \mathrm{IX}, \mathrm{VIII})$ [Miscellaneous Codex] 43,55

P. Bodmer XIII (+ P. Bodmer VII, XI, X, V, $\mathrm{XII}, \mathrm{XX}, \mathrm{IX}, \mathrm{VIII})$ [Miscellaneous Codex] 37 .n. 106, 40 Fig. 20, 55 
P. Bodmer XIV-XV 31 n. 89, 55

P. Bodmer XVI 31 n. 89, 56

P. Bodmer XVIII 31 n. 89, 56

P. Bodmer XIX 35, 36 + Fig. 17, 37, 56, 109 n. 220

P. Bodmer XX (+ P. Bodmer XII, XIII, VII, XI, $X, V, I X, V I I I)$ [Miscellaneous Codex] 31 n. 89,32 n. $93,35,37+$ n. 106, 40, 41 Fig. 21, 43, 55

P. Bodmer XXI (+ P. Chester Beatty Ac. 1389) 31 n. 89,35

P. Bodmer XXII (+ Grand Haven, Scriptorium, Mississippi Coptic Codex II) 56

P. Bodmer XXIII 31 n. 89, 32 n. 93, 56

P. Bodmer XXIV 31 n. 89, 34 Fig. 16, 35, 55

P. Bodmer XXV (+ P. Bodmer IV + P. Bodmer XXVI + P. Köln VIII 331+ P. Duke inv. 775) 31 n. 87, 32 n. 94, 35, 43, 45, 48 n. 121, 54, 56

P. Bodmer XXVI (+ P. Bodmer XXV + P. Bodmer IV + P. Köln VIII 331+ P. Duke inv. 775) $43,45,54,56$

P. Bodmer XXVII (+ P. Bodmer XLV-XLVIXLVII) 31 n. 89, 35, 55

P. Bodmer XXIX (+ P. Bodmer XXX-XXXVIII) [Codex Visionum] $23+$ n. 52, $31 \mathrm{n}$. 89, 35, 49, 51 Fig 30, 54, 56

P. Bodmer XXX-XXXVIII (+ P. Bodmer XXIX) [Codex Visionum] $23+$ n. 52, 24 n. 57, 54, 77

P. Bodmer XL 31 n. 89,56

P. Bodmer XLI 31 n. $89+91$

P. Bodmer XLV-XLVI-XLVII (+ P. Bodmer XXVII) 55

P. Bodmer LIV-LVI 32

P. Bouriant 1149

P. Bouriant 561 n. 142

P. Cairo 10759 134, 156 n. 365

P. Cairo 140 (+ P. Berol. inv. 5003, P. Gen. IV 158) 46

P. Cairo inv. $43227 \quad 136$

P. Cairo Isid. 232 n. 93

P. Chester Beatty 2026 (+ Oslo, Schøyen Collection, MS 193) 31 n. 89, 55

P. Chester Beatty Ac. 1389 (+ P. Bodmer XXI) 20,31 n. 89,35
P. Chester Beatty Ac. 149947 n. 120

P. Chester Beatty Ac. 2555 (+ P. Köln V 214, P. Bodmer II) 31 n. 89, 55

P. Chester Beatty IX-X 41

P. Col. XI 29378

P. Duke inv. 775 (+ P. Bodmer XXV + P. Bodmer IV + P. Bodmer XXVI + P. Köln VIII 331) 31 n. 89, 32 n. 92, 43, 56

P. Flor. II $108 \mathrm{r} 135$

P. Flor. II 108v 135

P. Gen. IV 158 (+ P. Cairo 140 + P. Berol. inv. 5003) 46

P. Genova 261 n. 142

P. Grenf. II 112 XIV

P. Hal.55A 78

P. Kellis Copt. 36111

P. Kellis I 26 XIV n. 23

P. Kellis I 61 XIV n. 23

P. Kellis Lit. II 97 XIII, XIV

P. Köln I 5239

P. Köln V 214 (+ P. Bodmer II + P. Chester Beatty Ac. 2555) 31 n. 89, 55

P. Köln VIII 331 (+ P. Bodmer XXV + P. Bodmer IV + P. Bodmer XXVI + P. Duke inv. 775) 31 n. 89, $32.92,43$, 56,155

P. Köln inv. 4780 [Mani-Codex] $138+$ n. $308,155,156,157+$ Fig. 67, $158+$ Fig 68

P. Lit. Palau Rib. 2061 n. 142

P. Lond. Copt. 5224

P. Lond. inv. 2852 (+ P. Ryl. III 489) 4

P. Lond. Lit. 33122

P. Lond. VI 1920 4, 32 n. 93

P. Mich. III 13223

P. Monts. Roca. inv. 128-178 (+ P. Monts Roca inv. 292, 338) 31 n. 89, 56

P. Monts. Roca. inv. 292 (+ P. Monts. Roca inv. 128-178, 338) $31 \mathrm{n} .89,32 \mathrm{n}$. 94, 47, 56

P. Monts. Roca. inv. 338 (+ P. Monts. Roca inv. 128-178, 292) $31 \mathrm{n} .87,32 \mathrm{n}$. $94,47,56$

P. Nag Hamm. $22 \quad 25$

P. Nag Hamm. 2325

P. Nag Hamm. 6225

P. Nag Hamm. 6325 
P. Nag Hamm. 6425

P. Nag Hamm. 6525

P. Ness. II 1149 n. 352

P. Ness. II 6150 Fig. 61, 151

P. Oxy. I 2239

P. Oxy. II 20939

P. Oxy. II 223135

P. Oxy. II 237135

P. Oxy. III 412 23, 35

P. Oxy. IV 66168

P. Oxy. IV 664 (+ P. Oxy. L 3544) 23

P. Oxy. VI 84878,94

P. Oxy. XIII 159594

P. Oxy. IX 117961

P. Oxy. LX 4047122

P. Oxy. LX 4051122

P. Oxy. LXII 4327 70, 71 Fig. 36

\section{Manuscripts}

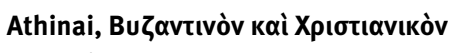

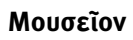

BХM 0862 [КПр 0225 ; Pallas, бп. 21] (+ Lerma, Castello Spinola, s.n. + London, BL Cotton Tit. C. XV + New York, The Pierpont Morgan Library, MS. 874 + Patmos, Movì toũ Ayíou

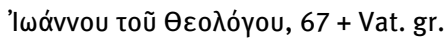
2305 + Sankt-Peterburg, Rossijskaia Nacional'naja Biblioteka, Petropol. gr. $537+$ Wien, ÖNB Theol. gr. 31, ff. 25-26 + Thessaloniki, 'Apxaı̀oүıxò


74 n. 171



gr. 57 203, 204

gr. 59179 Fig. 73

gr. 94205 n. 511

\section{Baltimore, The Walter Art Gallery}

W 520 201, 205 n.513

W 524198 n. 482
P. Oxy. LII 366384,122

P. Oxy. LIV 3746 IX

P. Oxy. LVI 3837 (+ P. Oxy. X 1250) 39

P. Oxy. LVI 385084

P. Oxy. LXVII 4608 IX n. 11, X

P. Oxy. LXVII $4611 X$

P. Oxy. LXIV 4441 IX

P. Oxy. LXXVI 5074147 Fig. 56

P. Oxy. XLIX 3509 71, 72 Fig. 37

P. Oxy. L 3544 (+ P. Oxy. IV 664) 23

P. Paris inv. E 667877

P. Raineri II 77,80

P. Vindob. G 29816a 84, 120, 121, 122

P. Whithouse 84

\section{Basel, Universitätsbibliothek}

A N III 12169

\section{Berlin, Staatsbibliothek}

Phillipps 1538198 n. 482, 199 n. 486, 202 Fig. $81+82,203,209$

\section{Cairo, Coptic Museum}

inv. 4851 [NHC III] 1 n. 1 + n. 4, 2, 4 + Fig.

$$
\text { 1, 5, } 28 \text { n. } 79
$$

inv. 10544 [NHC II] 2, 10, 12 Fig. 8, $13+n$. 30,17 n. $37,27,28$ n. 79,29 n. $86+$ n. 87,124 n. 273

inv. 10545 [NHC XIII] 2, 13 n. 30, 16, 17, 18

Fig. 13,18 n. 37,27 n. 76,29 n. 87

inv. 10546 [NHC VII] 1 n. 4, 2, 16 Fig. 11, $25+$ n. $69+$ n. 70,26

inv. 10547 [NHC XI] 1 n. 4, 2, 16 + Fig. 12, 18, 19 Fig. 15, 26, 27 n. 76,

inv. 10548 [NHC V] 1 n. 4, 6 Fig. 3, 7, 25, 124 n. 273

inv. 10549 [NHC VI] 7 Fig. 4

inv. 10550 [NHC VIII] 2, 8 Fig. 5

inv. 10551 [NHC X] 2, 10, 14 Fig. 9, 28 n. 81 
inv. 10552 [NHC IV] 2, 5 Fig. 2, 6, 7, 20, 28 n. $81+$ n. 84

inv. 10553 [NHC IX] 1 n. 4, 2, $9+$ Fig. 6 inv. $10554+10589+10590$ [NHC I] 1 + n. 1 + n. 4, 10, 11 Fig. 7, 18, 19 Fig. 14, 25 n. 70, 26, 27, 28 n. 79, inv. 10555 [NHC XII] 2, 10, 15 Fig. 10, 35

\section{Cambridge, University Library}

Cantabr. Add. 1875 74, 80

Cantabr. MS. T-S $12.184+20.50122$

\section{Città del Vaticano, Biblioteca Apostolica Vaticana}

Barb. gr. 310204 n. 508

Barb. gr. 336 79, 81, 95, 97

Barb. gr. 472 79, 81, 95, 97

Borg. Copto 109, cass. VII, fasc. 65, ff. $1-$ 21 (+ Paris, BNF Copte 132/2, f. 60 + Paris, BNF Copte $120 / 10$, f. $209+$ Paris, BNF Copte 129/9, ff. 49, 65, 76 + Paris, BNF Copte 128/8, ff. 121-122, 140, 157 + Paris, BNF Copte 129/7, f. 35 + New York, The Pierpont Morgan Library, MS. 664 A [4], ff. 1-2) 24

Borg. Copto 109, cass. VII, fasc. 6522

Vat. gr. 73199

Vat. gr. $351178+$ n. 430,180 n. 437

Vat. gr. 354 144, 145 Fig. 53, 160, 188, 190

Vat. gr. 357177

Vat. gr. 364199 n. 489

Vat. gr. 428 162, $164+$ n. 388

Vat. gr. 60464 n. 153

Vat. gr. 699 162, 164 n. 388

Vat. gr. 749159

Vat. gr. 1067194 n. 475

Vat. gr. 1209 [Vaticanus] 75, 76

Vat. gr. 1231208

Vat. gr. $128875,77,81,93,169$

Vat. gr. $145679,81,97$

Vat. gr. 1522166 n. 392, 178, 179 n. 432, 180 ,

Vat. gr. 1613198,199 n. 484, 202, 204, 205 n. 511

Vat. gr. 1615199 n. 489
Vat. gr. 1666 68, 79, 81, 95, 97

Vat. gr. 2061A, part A 75, 81, 82 n. 186, 93

Vat. gr. 2061A, part B 74, 81, 82, 93, 94

Vat. gr. 2061A, part $C \quad 74,80,82,93$

Vat. gr. 2106 (+ Marc. gr. Z. 1) $183+n$. 447, 184 n. 449, 190

Vat. gr. $2144 \quad 162,164$

Vat. gr. 2302 74, 81, 93, 94

Vat. gr. 2305 (+ Lerma, Castello Spinola,

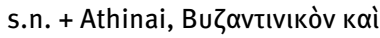

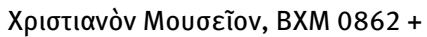
London, BL Cotton Tit. C. XV + New York, The Pierpont Morgan Library, MS. 874 + Patmos, Movì toũ Ayíou

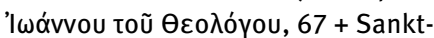
Peterburg, Rossijskaia Nacional'naja Biblioteka, Petropol. gr. 537 + Wien, ÖNB Theol. gr. 31, ff. 25-26 + Thessa-

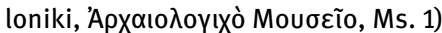
[Codex N of the Gospels] $74+$ n. 171, 80

Vat. gr. 2306 75, 77, 81, 93, 94

Vat. gr. 2591 77, 94

Vat. Ottob. gr. 4204 n. 508

Vat. Syr. 162 (+ London, BL Add. 14665)

[Z1] 75, 81, 82 n. 186,93

Vat. Syr. 162 (+ London, BL Add. 14665)

[Z2] 75, 81, 82 n. 186, 93

Vat. Syr. 162 (+ London, BL Add. 14665)

[Z4] 75, 81, 82 n. 186, 93

Vat. Syr. 162 (+ London, BL Add. 14665)

[Z6] 75, 81, 82 n. 184, 93

Vat. Urb. gr. 20198,199 n. 482

\section{El Escorial, Real Biblioteca}

T.III.3 (gr. 163) 208

Ф III 20161

$\Psi . I .14181$

\section{Firenze, Biblioteca Medicea Laurenziana}

Conv. Soppr. 152 79, 81

Conv. Soppr. 202 184, 185 Fig. 75, 190

Plut. 6.31 170, 177

Plut. 28.26 185, 186 Fig. 76

Plut. 70.3182

Plut. 81.11182 
Grottaferrata, Biblioteca della Badia Greca

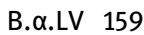

B. $\alpha$. LVI n. I [a] 79, 81, 97

Z.a.XXIV [b] 79, 81

\section{Hagion Oros}

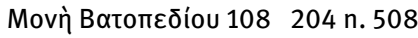

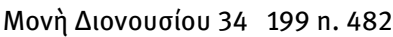

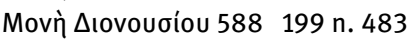

Movì 'Ißńpwv 1205 n. 516



Movì Meүíotns ^aúpas A 86193 n. 470

Movì Meүíotns ^aúpas A 92205 n. 514



Centrale BIbliotheek van de

Katholike Universiteit, fr. H. Omont

8) 74,80

\section{Iaşi, Biblioteca Centrală Universitară «Mihai Eminescu»}

Ms. 160 (IV-34) 178

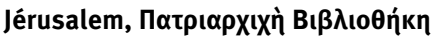

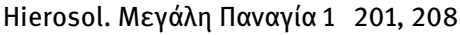

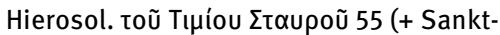

Peterburg, Rossijskaia Nacional'naja Bibliotheka, Petropol. gr. 339) 186 + n. 454,187 Fig. 77

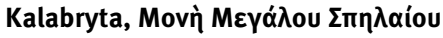

Ms. 1198 n. 482, 204 n. 508

Leiden, Bibliotheek der Rijksuniversiteit Leid. Voss. Gr. Q. 8 (+ Par. gr. 17, Petropol. RNB gr. 3) XIV, 122, 127

\section{Leipzig, Universitätsbibliothek}

gr. 1 (+ London, BL Add. MS. 43725 +

Sankt-Peterburg, Rossijskaia Nacional'naja Bibliotheka, Petropol. gr. 259 + Petropol. gr. 2 + Petropol. 0. 156 + Petropol. gr. $843+$ Sinā', Movì

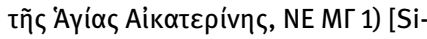
naiticus] $75,76,77$

gr. $7 \quad 142$ n. 328

\section{Lerma (Alessandria), Castello Spinola}

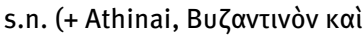

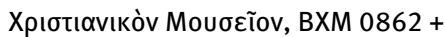
London, BL, Cotton Tit. C. XV + New York, The Pierpont Morgan Library, MS. 874 + Patmos, Movì toũ Ayíou 'I 2305 + Sankt-Peterburg, Rossijskaia Nacional'naja Biblioteka, Petropol. gr. 537 + Wien, ÖNB Theol. gr. 31, ff.



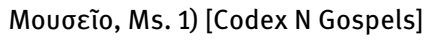
74 n. 171

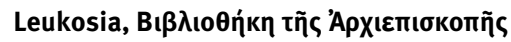
Kúmpou

Leukos. Ms. 25204 n. 508

Leuven, Centrale Bibliothek van de

Katholieke Universiteit

Copt. Lov. 9106 n. 215

fr. H. Omont 8 (+ Hagion Oros, Movì

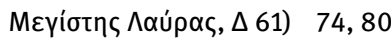

\section{London, British Library}

Lond. Add. 5111 73, 80

Lond. Add. 14665 (+Vat. Syr. 162) 75, 81, 82 n. 186,93

Lond. Add. 17210 122, 124

Lond. Add. 17211 (ff. 1-48) 76, 77, 79, 80, 93, 94, 96, 124

Lond. Add. 17211 (ff. 49-53) 80, 93, 94, 96,124

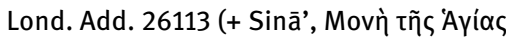

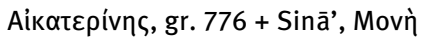


Lond. Add. 43725 (see also Leipzig, gr. 1; Sankt-Peterburg, Rossijskaia Nacional'naja Bibliotheka, Petropol. gr. $259+$ Petropol. gr. 2 + Petropol. 0.156 + gr. 843; Sinā', Movì tñ

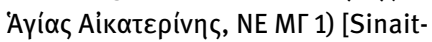
icus] $75,76,77$

Lond. Cotton Otho B.VI 78, 94

Lond. Cotton Tit. C. XV (+ Lerma, Castello Spinola, s.n. + Athinai, Bußаvtıvòv




$0862+$ New York, The Pierpont Morgan Library, MS. 874 + Patmos, Movì


+ Vat. gr. 2305 + Sankt-Peterburg, Rossijskaia Nacional'naja Biblioteka, Petropol. gr. $537+$ Wien, ÖNB Theol. gr. 31, ff. 25-26 + Thessaloniki,

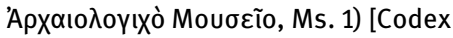
$\mathrm{N}$ of the Gospels] 74 n. 171

Lond. Harley 5598170

Lond. Orient. MS 9035 (13) 24

Lond. Orient. MS 14149 (13-27) 24

Lond. Royal MS. 1 D V-VIII [Codex Alexandrinus] 75

London, Lambeth Palace Library

MS. $1214 \quad 208+$ n. 528

Madrid, Biblioteca Nacional de España

Matrit. Res. 235207 n. 512

Messina, Biblioteca regionale universitaria Messin. F.V. 18 201, 208

Milano, Biblioteca Ambrosiana

Ambr. B 114 sup. 183 n. 445

Ambr. E 49-50 inf. 159

Ambr. L 99 sup. 134

Modena, Biblioteca Estense Universitaria

Mut. gr. 73 ( $\alpha . W .2 .6) \quad 79,81,95,97$

Moskva, Gosudarstvennyi Istoričeskij

Muzei

Synod. gr. 57 (Vlad. 139) 182

Synod. gr. 96 (Vlad. 98) 199, 204 n. 510

Synod. gr. 99 (Vlad. 99) 204 n. 508

Synod. gr. 313 (Vlad. 185) 204 n. 510, 208

\section{Moskva, Rossijskaja Gosudarstvennaja}

Biblioteka

Ф 201(Sobranie rukopisej A.S. Norova), 18, 1 144 n. 333

München, Bayerische Staatsbibliothek Monac. Clm 777564 n. 153
Monac. lat. 29022e 79, 81, 95, 97

Napoli, Biblioteca Nazionale «Vittorio Emanuele III»

ex Vindob. gr. 1 79, 81, 95, 97

ex Vindob. gr. 2 73, 80, 93

New York, The Pierpont Morgan Library MS. 664 A (4), ff. 1-2 (+ Paris, BNF Copte $129 / 7$, f. $35+$ Copte $128 / 8$, ff. 121122, 140, $157+$ Copte 129/9, ff. 49, $65,76+$ Copte $120 / 10$, f. $209+$ Copte 132/2, f. $60+$ Vat. Borg. Copt. 109, cass. VII, fasc. 65, ff. 1-21) 24

MS. 874 (+ Lerma, Castello Spinola, s.n. +

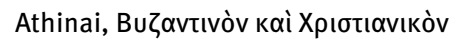

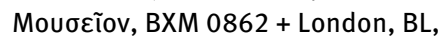
Cotton Tit. C. XV + Patmos, Movì toũ

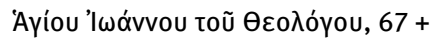
Vat. gr. 2305 + Sankt-Peterburg, Rossijskaia Nacional'naja Biblioteka, Petropol. gr. $537+$ Wien, ÖNB Theol. gr. 31, ff. 25-26 + Thessaloniki,


N Gospels] 74 n. 171

\section{Oxford, Bodleian Library}

Oxon. Auct. E. 2. 12208

Oxon. Auct. T. inf. II. 2 (+ Sankt-Peterburg, Rossijskaja Naciolal'naja Biblioteka, gr. 33) 158 n. 360

Oxon. Auct. T. inf. II. $6201+$ Fig. 80, 204 n. 508

Oxon. Canon. gr. 92 179, 200

Oxon. Canon. gr. 110198 n. 482, 199 n. 486, 202, 204 n. 508, 209

Oxon. Laud. gr. 35 79, 81, 95, 97

Oxon. Laud. gr. 75 188, 189 Fig. 78

Paris, Bibliothèque nationale de France

Coislin 1169

Coislin $31200+$ n. 493, 205 n. 516

Coislin 46204 n. 508

Coislin 186 79, 81, 95, 97

Copte 120/10, f. 209 (+ Paris, BNF Copte 129/9, ff. 49, 65, 76 + Copte 128/8, ff. 121-122, 140, $157+$ Copte 129/7, f. 
$35+$ New York, Pierpont Morgan Library, 664 A (4), ff. 1-2 + Paris, BNF Copte 132/2, f. 60; Vat. Borg. Copt. 109 , cass. VII, fasc. 65, ff. 1-21) 24 Copte 128/8, ff. 121-122, 140, 157 (+ Paris, BNF Copte 129/7, f. 35; New York, Pierpont Morgan Library, 664 A (4), ff. 1-2; Paris, BNF Copte 129/9, ff. 49, 65, 76 + Copte 120/10, f. $209+$ Copte 132/2, f. 60; Vat. Borg. Copt. 109 , cass. VII, fasc. 65, ff. 1-21) 24 Copte 129/7, f. 35 (New York, The Pierpont Morgan Library, 664 A (4), ff. 1-2 + Paris, BNF Copte 128/8, ff. 121-122, 140, 157 + Copte 129/9, ff. 49, 65, 76 + Copte 120/10, f. $209+$ Copte 132/2, f. $60+$ Vat. Borg. Copt. 109, cass. VII, fasc. 65 , ff. 1-21) 24

Copte 129/9, ff. 49, 65, 76 (+ Paris, BNF Copte 128/8, ff. 121-122, 140, $157+$ Copte 129/7, f. 35 + New York, Pierpont Morgan Library, 664 A (4), ff. 1-2 + Copte 120/10, f. $209+$ Copte $132 / 2$, f. $60+$ Vat. Borg. Copt. 109, cass. VII, fasc. 65, ff. 1-21) 24 Copte 132/2, f. 60 (+ Paris, BNF Copte $120 / 10$, f. $209+$ Copte 129/9, ff. 49, $65,76+$ Copte $128 / 8$, ff. 121-122, 140, $157+$ Copte 129/7, f. $35+$ New York, Pierpont Morgan Library, 664 A (4), ff. 1-2 +Vat. Borg. Copt. 109, cass. VII, fasc. 65 , ff. 1-21) 24

Paris. gr. 9124 n. 272

Paris. gr. 17 (+ Leiden Voss. gr. Q. 8 + Sankt-Peterburg, Rossijskaja Naciolal'naja Biblioteka, gr. 3) 122, 127 Paris. gr. 70198 n. 482, 204 n. 508, 209 Paris. gr. $107+107 \mathrm{~A}+107 \mathrm{~B}$ 79, 81, 95, 97 Paris. gr. 134208

Paris. gr. 139 199, 204 n. 508, 209

Paris. gr. 146199

Paris. gr. 230198 n. 483

Paris. gr. 277174 n. 418

Paris. gr. 278168 n. 389, 178 + Fig. 72, 179 n. 433,180 n. 437

Paris. gr. 437 151, 152 Fig. 62, 157

Paris. gr. 480204 n. 508
Paris. gr. 510152 + Fig. 69, 159, 161, 163

Paris. gr. 629199 n. 489

Paris. gr. 676204 n. 508

Paris. gr. 713 (+ Paris, BNF Suppl. gr. 240) 204 n. 508

Paris. gr. 923152 Fi. 63, 153, 154, 159

Paris gr. 1419199 n. 489

Paris. lat. 1188464 n. 153

Paris. Suppl. gr. 240 (+ Paris, BNF gr. 713) 204 n. 508

Paris. Suppl. gr. 905 79, 81, 95, 97

Paris. Suppl. gr. 1155 79, 81, 95

Paris. Suppl. gr. 1262208 n. 526

Paris. Suppl. gr. 1286 74, 80, 93, 169 n. 401

\section{Patmos, Movì toũ Ayíou 'Iwávvou toũ

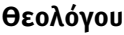

Patm. 43204 n. 508

Patm. 44204 n. 508

Patm. 67 (+ Lerma, Castello Spinola, s.n.

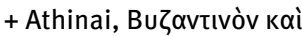

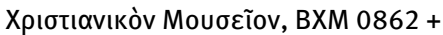
London, British Library, Cotton Tit. C. $\mathrm{XV}+$ New York, The Pierpont Morgan Library, MS. 874 + Vat. gr. 2305 + Sankt-Peterburg, Rossijskaia Nacional'naja Biblioteka, Petropol. gr. 537 + Wien, ÖNB Theol. gr. 31, ff. 25-26 + Thessaloniki, 'Apx


pels] $74 \mathrm{n} .171$

Patm. 70174 n. 418

Patm. 171 79, 80, 81, 95, 97

Roma, Biblioteca Vallicelliana

Vallic. Ms. C 34/IV 79, 81, 97

\section{Rossano Calabro, Museo Arcivescovile}

s.n. [Codex Purpureus Rossanensis] 74, 80, 93, 96, 169 n. 401

\section{Sankt-Peterburg, Rossijskaia Nacion- al'naja Biblioteka}

Petropol. gr. 2 (+ London, BL, Add. MS. 43725 + Leipzig, gr. 1+ SanktPeterburg, Rossijskaia Nacional'naja 
Bibliotheka, Petropol. gr. $259+$

Petropol. 0. 156 + Petropol. gr. $843+$



МГ 1) [Sinaiticus] 75, 76, 77

Petropol. gr. 3 (+ Leiden Voss. Gr. Q. 8 + Paris, BNF gr. 17) [Sarravianus] 122, 127

Petropol. gr. 12 (+ Sankt-Peterburg, Rossijskaja Naciolal'naja Biblioteka, gr. 278 + Sankt-Peterburg, Rossijskaja Akademija Nauk Biblioteka, Dmitrie-

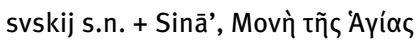

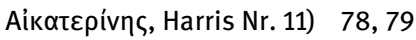

Petropol.gr. 21+21a 176

Petropol. gr. 33 (+ Oxford, Bodlean Library, Auct. T. inf. II. 2) 159 n. 368

Petropol. gr. 216 (+ Sinā', Movì tñ s Ayías Аікатврі́vпৎ, NE МГ 33) 143 Fig. 52, 144, 159

Petropol. gr. 258A (+ Sinā', Movì tñ

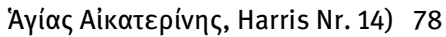

Petropol. gr. 259 (+ London, BL, Add. MS. 43725 + Leipzig, gr. 1 + SanktPeterburg, Rossijskaia Nacional'naja Bibliotheka, Petropol. gr. $2+$ Petropol. $0.156+$ Petropol. gr. $843+$

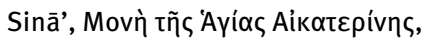
MГ 1) [Sinaiticus] 75, 76, 77

Petropol. gr. 262144 n. 333

Petropol. gr. 283 (+ Sinā', Movì tñ s 'Ayías Aikatepívns, gr. 213) 144, 262 n. 333

Petropol. gr. 339 (+ Jérusalem,

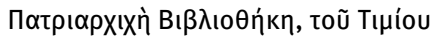

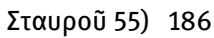

Petropol. gr. 537 (+ Lerma, Castello Spino-

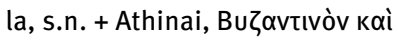

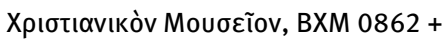
London, BL, Cotton Tit. C. XV + New York, The Pierpont Morgan Library, MS. 874 + Patmos, Movì toũ Ayíou

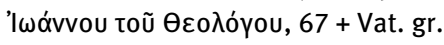
$2305+$ Wien, ÖNB Theol. gr. 31, ff. 25-26 + Thessaloniki, Apxaı̀oyıxò Mouбeĩo, Ms. 1) [Codex N of the Gospels] $74 \mathrm{n} .171$

Petropol. gr. 843 (+ London, BL, Add. MS. 43725 + Leipzig, gr. 1 + Sankt-
Peterburg, Rossijskaia Nacional'naja Bibliotheka, Petropol. gr. $259+$ Petropol. gr. $2+$ Petropol. 0. $156+$

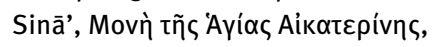
MГ 1) [Sinaiticus] 75, 76, 77

Petropol. 0. 156 (+ London, BL Add. MS. 43725 + Leipzig, gr. 1 + SanktPeterburg, Rossijskaia Nacional'naja Bibliotheka, Petropol. gr. $259+$ Petropol. gr. $2+$ Petropol. gr. $843+$


MГ 1) [Sinaiticus] 75, 76, 77

\section{Sankt-Peterburg, Rossijskaja Akademija Nauk, Biblioteka \\ RAIK 194 (+ Sinā', Movì tñ 'Aүías

 Harris App. 16, 22) 143}

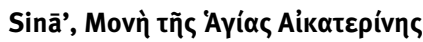

Sin. ar. 116 144, 146 Fig. 55, 161

Sin. gr. 33144 n. 333

Sin. gr. 204198 n. 482, 203, 205 n. 516

Sin. gr. 210 (+ Sinā', Movì tñ s 'Aүías

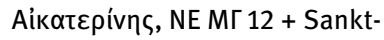
Peterburg, Rossijskaja Akademija Nauk, Biblioteka, RAIK 194 + Sinā',

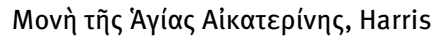
App. 16, 22) 68, 143 + Fig. 51

Sin. gr. 213 (+ Sankt-Peterburg, Rossijskaja Naciolal'naja Biblioteka, gr. 283) 144, 145 Fig. 54

Sin. gr. 22179

Sin. gr. 491161

Sin. gr. 497205 n. 514

Sin. gr. 776 (+ London, BL Add. 26113 +


gr. 1593) 124 n. 289

Sin. gr. 1593 (+ London, BL Add. 26113,

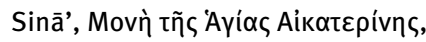
gr. 776) 134 n. 289

Sin. Harris App. 16, 22 (+ Sankt-Peterburg, Rossijskaja Akademija Nauk, Biblioteka, RAIK $194+$ Sinā', Movì tñ

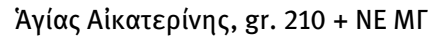
12) 143 
Sin. Harris Nr. 11 (+ Sankt-Peterburg, Rossijskaja Naciolal'naja Biblioteka, gr. 12 + gr. 278 + Sankt-Peterburg, Rossijskaja Akademija Nauk Biblioteka, Dmitriesvskij s.n.) 78, 79, 94

Sin. Harris Nr. 14 (+ Sankt- Peterburg, Rossijskaja Naciolal'naja Biblioteka,gr. 258A) 78, 79

Sin. NE MГ 1 (+ London, BL Add. 43725 + Leipzig gr. $1+$ Sankt-Peterburg, Rossijskaia Nacional'naja Bibliotheka, Petropol. gr. 259 + Petropol. gr. $2+$ Petropol. 0. $156+$ Petropol. gr. 843) [Sinaiticus] 75, 76, 77

Sin. NE MГ 12 (+ Sinā', Movì tñ ऽ 'Aүías


Sankt-Peterburg, Rossijskaja Akademija Nauk, Biblioteka, RAIK 194 +

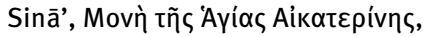
gr. 210) 68, 69 Fig. 34, 143, 146

Sin. NE MГ 1478

Sin. NE MГ 33 (+ Sankt-Peterburg, Rossijskaja Naciolal'naja Biblioteka, gr. 216) $144+$ n. 333, 159

Sin. NE МГ 46161

Sin. NE MГ 51161

Sin. NE MГ $70 \quad 78,79$

Sin. NE MГ 7178

Sin. NE MГ $76 \quad 78,79$

Sin. NE MГ 78161

Sin. NE MГ 8761 n. 142, 78, 79

Sin. NE MГ 10778

Sin. Politis 1b 78, 79, 94

Sin. syr. 30151

Sofija, Naučen Centr'r za slavjanovizantijski proučvanja «Ivan Dujčev» k'm Sofijskija Universitet «Kliment Ochridski»

gr. 387176

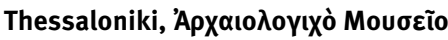

Ms. 1 (+ Lerma, Castello Spinola, s.n.; +

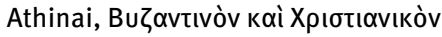

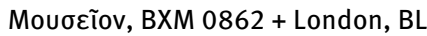
Cotton Tit. C. XV + New York, The Pierpont Morgan Library MS. 874 +
Patmos, Movì toũ Ayíou 'I $\omega$ ávvou toũ


Sankt-Peterburg, Rossijskaia Nacional'naja Biblioteka, Petropol. gr. 537 + Wien, ÖNB Theol. gr. 31, ff. 25-26 [Codex N Gospels] 74 n. 171

\section{Tiranë, Arkivat e Shtetit}

Berat. 1 74, 80, 93, 96

Torino, Biblioteca Nazionale Universitaria Taurin. B II 22176 + Fig. 70, 177 + Fig. 71, 179

\section{Venezia, Biblioteca Nazionale Marciana}

Marc. gr. Z. 1 (+ Vat. gr. 2106) $183+n$. $447+$ n. 449, 184 Fig. 74,190

Marc. gr. Z. $360203+$ Fig. 83 + Fig. 84, 204 n. 508, 209

Marc. gr. Z. 454182

Marc. gr. Z. 53199 n. 489

Marc. gr. 18 73, 80, 93, 198 n. 482, 201, 205 n. 515, 208

Marc. gr. I 18 93, 201, 204 n. 508, 209

Marc. gr.I 45171

Marc. lat. I 100193 n. 469

Washington, Smithsonian Institution

Freer Gallery, Cod. Freer 1 (06.292) 76, $77,80,94,96,122,124$

Freer Gallery, Cod. Freer 4 (06.275) 76, $77,79,80,94,96,121,122,122,124$ + n. 272

Freer Gallery, Cod. Freer copt. 124 Freer Gallery, Cod. Freer W (06.274) 135 + n. 292, 139, $155+$ Fig. 65, 156 + Fig. $66+$ n. $365,157,161$

Wien, Österreichische Nationalbibliothek Vindob. lat. 954 79, 81, 95, 97

Vindob. Med. gr. 1 68, 73, 79, 80, 93, 148 + n. 346, 149 Fig. 57-59, 155, 157, 161

Vindob. Suppl. gr. 50* 198 n. 482

Vindob. Suppl. gr. 52198 n. 482

Vindob. Suppl. gr. 121 79, 81, 95

Vindob. Suppl. gr. 122200 
Vindob. Theol. gr. 31, ff. 1-24 79, 80, 93, 96,169

Vindob. Theol. gr. 31, ff. 25-26 (+ Lerma, Castello Spinola, s.n. + Athinai,



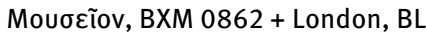
Cotton Tit. C. XV + New York, The Pierpont Morgan Library, MS. $874+$ Patmos, Movì toũ Ayíou 'I


Sankt-Peterburg, Rossijskaia Nacional'naja Biblioteka, Petropol. gr. 537 + Thessaloniki, 'Apxaı
Mouбeĩo, Ms. 1) [Codex N Gospels] 74 n. 171

Vindob. Theol. gr. 240 203, 209

Wolfenbüttel, Herzog August Bibliothek Guelferb. 75a Helmst 73, 79, 80, 93 Weissemb. gr. 64, part I $79,81,95,97$ Weissemb. gr. 64, part II 79, 81, 95, 97

Zürich, Private collection NussbergerTchacos

Codex Tchacos [Gospel of Judas] XIII, XIV, 124, 125 Fig. 49, 126 Fig. 5

\section{Repertorium}

Schüssler, Biblia Coptica

sa 9116 + Fig. 46

sa 14107

sa 15110,111

sa 19117

sa 30115 Fig. 30, 116

sa 35108,109

sa 48108,109

sa 49108 + Fig. 41, 109 + Fig. 42

sa 53119

sa 57111

sa 60105

sa 61114

sa 64 114, 115 Fig. 44

sa 78112

sa 80117

sa 95114

sa 98117

sa 109119

sa 111117

sa 117 ex 105

sa 120106,107 Fig. 40

sa 122128

sa 123128

sa 125128

sa 126128

sa 133128

sa 135128

sa 141128

$\begin{array}{ll}\text { sa } 142 & 128 \\ \text { sa } 144 & 128 \\ \text { sa } 150 & 128 \\ \text { sa } 157 & 128 \\ \text { sa } 160 & 128 \\ \text { sa } 163 & 128 \\ \text { sa } 167 & 128 \\ \text { sa } 170 & 128 \\ \text { sa } 171 & 128 \\ \text { sa } 179 & 128 \\ \text { sa } 183 & 128 \\ \text { sa } 184 & 128 \\ \text { sa } 187 & 129 \\ \text { sa } 189 & 129 \\ \text { sa } 193 & 129 \\ \text { sa } 195 & 129 \\ \text { sa 202 } & 129 \\ \text { sa 203 } & 129 \\ \text { sa 205 } & 129 \\ \text { sa 206 } & 129 \\ \text { sa 208 } & 129 \\ \text { sa 213 } & 129 \\ \text { sa 221 } & 129 \\ \text { sa 223 } & 129 \\ \text { sa 228 } & 129 \\ \text { sa 231 } & 129 \\ \text { sa 239 } & 129 \\ \text { sa 241 } & 129 \\ \text { sa 250 } & 129\end{array}$




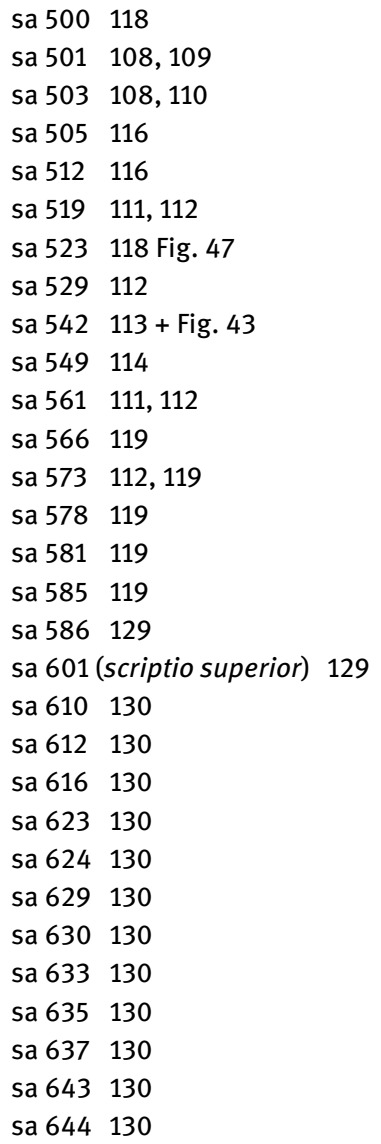




